\title{
Talking about routines in the field: the emergence of organizational capabilities in a new cellular phone network company.
}

Alessandro Narduzzo 1 , Elena Rocco ${ }^{2}$, Massimo Warglien 3

1 Computable and Experimental Economics Laboratory (CEEL), University of Trento 2 University Ca' Foscari, Venezia and University of Michigan, School of Information 3 University Ca' Foscari, Venezia and CEEL, University of Trento 


\section{Introduction: unbundling routines}

To many students of organizational life, part of the attractiveness of the routine concept lies in its capacity to tie together familiar features of how organizations do and know how to do things. Rather than being defined once-for-all, the concept of organizational routine is progressively circumscribed by the set of properties one learns to associate to it. A familiar list of such properties would include adjectives like tacit, automatic, repetitive, distributed, situated, rule-based, political. Attempts to provide an unambiguous definition of routines have proved to be only mildly successful, and Sid Winter has legitimately claimed that the polisemy of the concept is part of its strength (Winter 1986; Cohen et al. 1996). In fact, the concept of routines is caught in a fancy recursion, since it reproduces at a higher level part of the features it describes. It is somehow tacit, since there is more in the concept than one can say (and people learn it mostly through examples); it is distributed in the community of its users and, in some cases, it is a truce among the different and sometimes conflicting claims students make on the definition of organizational capabilities (see again Cohen et al. 1996).

Thus, the concept of routine looks like a bundle of features, among which users can emphasize those that better fit the specific aims and context of their analysis. However, what in most cases is a point of strength, turns out to be more problematic when one deals with case studies in the field. Being samples of one, case studies tend to emphasize the descriptive and interpretive accuracy of concepts, rather than their ability to capture recurring features across multiple experiences. Thus, when dealing with the field, some reverse engineering is needed and unbundling concepts becomes the dominant strategy.

This is what this chapter is mostly about. It is a case study in the emergence of organizational capabilities in a new born cellular phone company. The case study looks at how competencies are accumulated in the key activities of network installation and maintenance in the first year of operations. During such year, we have been tracking the capabilities building process through multiple data gathering activities, including interviews, systematic screening of internal documents, and a six months presence in the field with two network technicians' teams by one of us. Inevitably, compressing in a few pages the huge amount of (often unstructured) information such observations have generated implies some subjective selection and the usual tradeoffs among accuracy, generality and simplicity (Weick 1979). In doing so, we have been guided by a twofold concern. On the one hand, we have tried to understand whether the concept of routine provides a suitable framework for describing and interpreting the emergence of organizational capabilities, and how the concept should be articulated to account for our observations; on the other one hand, we have tried to learn from the field some lessons 
on the nature of organizational routines. Thus, our chapter can be better framed as a "conversation with the field" about routines, a round trip between concepts and data.

While sections are mostly empirical, each one contains a "wrapping up" paragraph where most conceptual implications of our findings are summarized.

After a short presentation of the field setting (section 2), we focus on four key processes related to capabilities building. Section 3 reconstructs some key features of organizational imprinting, or the original accumulation of a stock of capabilities in the early months of organizational life. Section four deals with how technicians learn to fix troubles, looking into the way individual skills and collective capabilities interweave. Section five describes the emergence of network installation routines, calling in processes of emergent division of labor and the recombination of elementary routines in more complex patterns of action. Section six looks at the evolution of coordination within technicians' teams, reconstructing the emergence of hierarchical roles. Finally, we summarize what we have learned from the field.

\section{A case study: network technicians in a new cellular phone company}

Omnitel Pronto Italia (henceforth OPI) provides a service of wireless communication through cellular phones in Italy. By the time of this study, OPI was a just born company which has been in the market since the end of 1995. The organization is decentralized and physically distributed in the country. There are four zones covering four different geographical areas coordinated by the Head Quarters located in one of the four zones.

The first year activity has been extremely intensive and in a few months OPI has created a nationwide $\mathrm{GSM}^{1}$ network. Over 600 transceiver stations have been installed in less than six months (see figure 1).

\footnotetext{
${ }^{1}$ GSM (Global System of Mobile Communication) is the European standard technology for digital cellular communication.
} 


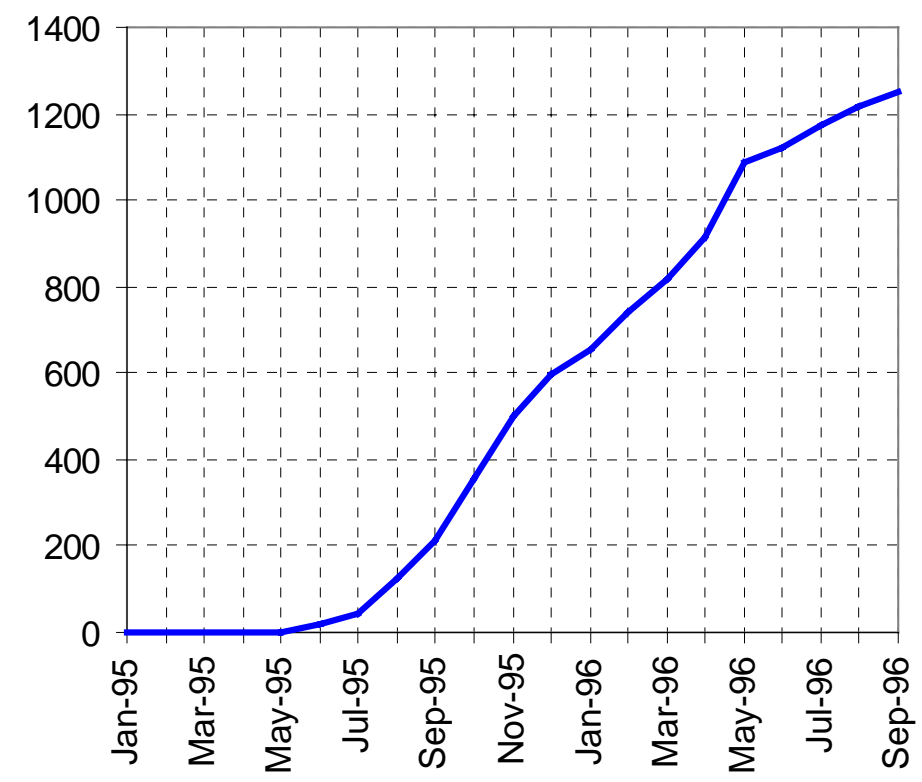

Figure 1. Number of BTS stations installed

OPI has performed a rapid growth to meet the legal requirements that define the minimal network dimension. Such a quick growth has generated heavy pressures on competence accumulation, especially with regards to competencies which are less known and understood. Among them, network technical competencies have high strategic relevance, meeting current definitions of organizational capabilities (Amit and Schoemaker 1993; Barney 1991; Teece 1984). Such competencies mainly refer to installation and maintenance activities. The capacity to rapidly install new stations is needed for both legal and marketing reasons. On the one hand, it allows to meet the national legal threshold for becoming authorized providers of cellular communication services; on the other hand, the geographic area of network coverage is a key parameter of service quality to customers' eyes. A second key technical competence is network maintenance: a high service reliability is considered by OPI a source of competitive advantage.

Installation and maintenance are complex tasks accomplished by a large number of organizational units and sub-units. Different units plan which parts of the country have to be covered, how many transceiver stations (called BTS ${ }^{2}$ stations) they need, where the stations should be placed and which configuration has to be chosen. Once the locations are identified they rent the space and build the sites. When the room is ready the GSM transceivers are installed and connected to the network. These last steps are followed by the so-called BTS specialists; they are the technicians in charge for the

\footnotetext{
${ }^{2}$ BTS stands for Base Transceiver Station and is the radio unit of the GSM infrastrucure that exchanges signals with cellular phones.
} 
maintenance of the network. In a few months OPI has recruited over one hundred skilled repairmen as BTS technicians. Most of them had previous experience with telecommunication technology (e.g. wireless communication, radio links), but none was familiar with the digital cellular GSM adopted by OPI.

BTS technicians are grouped in teams which are responsible for network activities over well defined areas. The main activities on BTS stations are: installation, trouble fixing and periodical maintenance. They have to handle a variety of problems and situations concerning the telecommunication system, electric (e.g. batteries) and electronic (e.g. smoke detectors) devices, and the air conditioning system. Taking care of BTS stations and the GSM network over a geographical area requires to monitor communication traffic, to make periodical checks and clean the sites, to upgrade hardware and software configurations, and to document the activity. BTS technicians have also close relationships with a variety of different external suppliers.

BTS technicians are organized in quasi-independent teams. These teams are spatially distributed and they are the most decentralized units of the company. Technicians' daily work follows a weekly schedule. The team's scheduling often changes during the week because of unexpected events such as alarms in the BTS stations which require immediate solutions.

During the first months BTS technicians belonging to the same team used to work together; later on, as the network size has increased, they started spreading around and working alone. Increasing physical isolation made face-to-face interaction a more rare event and the BTS technicians began to use their cellular phones as the main communication device to keep in touch and coordinate with others.

BTS technicians spend most of their time in the field, and some of them go to the office only once or twice a week. When they are in the field they (a) participate to the installation phase to build new BTS stations, (b) carry out the maintenance activity and (c) fix troubles when BTS alarms turn on. In fact, the BTS technicians may receive phone calls from other colleagues who monitor the whole network.

\section{Organizational imprinting: the "primitive accumulation" of capabilities}

The initial phase of OPI's activity has been thus characterized by at least two key constraints. First, there has been an urgency to reach a territorial network coverage sufficient to meet the deadline imposed by the National Telecommunication Department. That deadline marks the conclusion of the set-up phase and the opening of the full-fledged working phase. The second constraint has been the need to provide customers a regular and reliable service since the first day of the working phase. This implies that OPI had to reach a critical threshold of competence within a short time 
span, running the new technology, concentrating learning, testing and trouble-shooting in a few months. This process of "primitive accumulation" of capabilities had to occur in the absence of direct feedback from fully operating conditions, in what was commonly referred to as a "simulated" working environment. Thus, most learning had to resort to substitutes of experience (Weick 1991) rather than to direct experience.

Besides knowledge and technology supplied by providers, the initial pool of resources needed to create OPI own operating capabilities were brought in recruiting skilled people coming from pre-existing organizations. Of course, it was impossible to buy such capabilities already packaged; therefore, OPI had to accumulate knowledge by hiring people from a host of "neighbor" organizations in the telecommunication industry (i. e. Telecom, Ericcson, Italtel, Bell Atlantic). All those people were bringing in different individual skills and practices from different systems of organizational routines and mapping them onto the new context. As a consequence, organizational learning initially occurs mainly through the "import" and replication of others' experience (vicariuos learning) rather than through direct one.

In the new, rapidly growing firm, field practice is to a large extent a space to fill, only partially structured by organizational policies, where people can recognize familiar cues and transfer their own past experience. For example, the use of maps is widespread in radio frequency activities. BTS technicians with previous jobs in radio frequency draw maps for supporting their trouble diagnostic activity, while others use text base information. Similarly, people with former experience in the domain of power stations tend to introduce new procedures for maintenance inspired by their previous job. Thus, the initial stock of competencies looks like an organizational patchwork, an assembly of heterogeneous elements borrowed from the environment.

Of course, some coherence needs to be conferred to such collection of practices. The key role in shaping more ordered patterns of action has been played by the zone technical supervisors, who have been hired among the first and have been in charge to recruit the technicians' teams and shape their local organization. However, since supervisors of different zones come from different organizations, each zone has developed an idiosyncratic work style. For example, a zone (that we will label "zone A") tends to reproduce the technocratic style of the organization its supervisor comes from. This means that trouble-fixing is conceived as a highly standardizable activity, and most efforts are devoted to creating a detailed set of standard operating procedures to deal with each possible trouble. The process of generating such procedures is inherited from the source organization of the supervisor, and often single procedures use SOPs from such organization as templates. Technicians are trained as executors of such procedures, and coordination is bureaucratic and rule-based. Conversely, in another zone ("zone B") the supervisor brings from its original organization a conception of trouble fixing as a complex task which requires reflection and diagnostic reasoning. In zone $\mathrm{B}$, the accent is on shared information rather than standard procedures, and efforts 
are devoted to creating databases and technical notes that can support such an information sharing process. The structure of data bases and technical notes is directly imported from the supervisor's previous experience. Coordination is informal and based on mutual adjustment.

Such local heterogeneity of practices is not surprising in a new, decentralized organization. What matters here is that those imported practices and the system of routines they generate tend to persist in spite of pressures for organization-wide homogeneity from the Head Quarters, and create an "organizational imprinting effect" that gives each zone a peculiar style and organizational flavor. Still now people easily identify the source of such flavor (e.g., it is common to refer to a given zone as being "Telecom style")

There are multiple reasons for such local persistence of early routines. A first reason is simply sunk costs. Once a given routine has generated investments in diagnostic software, formal procedures, databases, training and so forth, it creates economic and psychological inertia (Staw and Ross 1987). Sometimes sunk costs are strategically exploited by zones: e.g. committing skills and investing in costly software systems that support a given set of procedures makes less likely that Head Quarters will change such procedures.

A second reason is complementarities. Single routines do not stand alone, but usually are complementary to others. For example, the way a trouble is fixed has relationships with how troubles are communicated, how jobs are scheduled, how databases are built and updated and how past troubles are documented. Once a more or less coherent system of routines is installed, it cannot be changed piecemeal without damaging the global performance - it is trapped in a local optimum, to use a mathematical metaphor (Levinthal and Warglien 1997). Consequently, complementarities protect single routines from change pressures and defer change to major restructurings that often never come, because they are too costly, risky and disruptive.

There are also insulation factors that preserve localism and inhibit the convergence in practice among zones. One obvious insulation factor is space. Spatial proximity is a fundamental facilitator of processes of imitation and mutual learning; it favors communication, trust, and generates opportunities for joint work. Individuals who work on stations significantly distant from others, rarely meet team members, have to rely mostly on their own direct experience and develop idiosyncratic, often poor skills - for example, they develop too simplistic diagnostic models of troubles (see $\S 4$ ).

However, distance among individuals does not depend only on geographical space, but also on organizational structure, that affects communication opportunities and creates a sense of belonging to a same social unit. For example, interactions between 
Naples and Palermo, that are in the same zone, are more frequent than interactions between Naples and Rome, that belong to different zones, although the geographical distance among the latter is lesser than the one between the former. Indeed, both geographical and organizational distance seem to matter in our case. Interactions and exchange of experiences are more frequent within zones but to a lesser extent also across zones that are closer in space.

Time also matters. As different zones have started the set-up of the network in different moments, time delays play against the diffusion of local competencies. Since zones have not started at the same time, people do not simultaneously face the same problems and inter-zone meetings are often considered as a waste of time. This fact is also strategically used as an excuse to apply local solutions to common problems and intentionally avoid exchange of solutions among zones. For instance, during inter-zone meetings zone managers talk about problems they face, but they do not share and discuss solutions. As a result, there are indeed rare examples of imitation of solutions earlier developed in other zones.

However, distance in space, organizational structure and time act as insulation mechanisms also because there are powerful incentives to keep low levels of inter-zone learning and diffusion. Some competition among geographical units is commonplace in any decentralized organization. However, in our case Head Quarters have pushed competition among zones to improve zones' performance, especially with regards to effectiveness in setting up the network. The side effect is that the diffusion of best practices is inhibited - each zone has an incentive to perform better than the other ones.

This kind of strategic behavior is not only visible in the relations among zones, but also in the relations between each zone and the Head Quarters. Head Quarters have an interest to drive a process of convergence of work practices and documentation, in order to foster diffusion of best practices and keep monitoring and control of zone activities. On the converse, zones tend to preserve autonomy, maintaining local coherence. In the context of new organization building, this often implies the search for "first mover advantages" in the establishment of work practices. In other words, zones tend to anticipate Head Quarters in establishing procedures and documentation, tayloring such elements to their emergent routines. For example, Head Quarters have been developing a system that had to keep together the nation-wide monitoring of network troubles, the dispatching of trouble fixing tasks to technicians, and the recording of trouble histories. However, only the network monitoring activity and the dispatching to teams (not to team members) has been immediately available. In the meanwhile, some zones have developed their own parallel dispatching and trouble history recording systems. This implies that different zones have now different systems, making standardization increasingly difficult and leaving the key control functions in the local hands (for example, reliably assessing the average trouble fixing time is now possible only at the local level). Of course, the development of local systems is 
motivated by operating needs and the urgency to have key information without waiting for the full development of the central system. The point is that those local systems are developed without searching for an ex-ante agreement with Head Quarters and other zones on procedural and technological standards, thus creating the premises for ex-post incompatibility with low reversibility.

\section{Wrapping up}

Observing a new born organization with rapid growth rates provides a unique opportunity to analyze the nature of organizational imprinting (Stinchcombe 1965; Ginsberg and Baum 1994).

We have stressed that vicarious learning plays a fundamental role in performing the "primitive accumulation" of OPI's organizational capabilities. Having no time to resort to direct experience, the firm has found her competencies in the environment. The process of assimilation of such (heterogeneous) competencies has stressed local coherence versus firm-level integration and has generated within zones patterns of action that, once established, have proved stable and resilient to efforts of firm-wide standardization.

The local stabilization of initial capabilities is the key feature of the imprinting process. Our case study suggests that the process has a dual nature (Coriat e Dosi 1994). On the one hand, there are "functional" reasons supporting the stability in each zone of the initial core of organizational practices: urgency, sunk costs, complementarities among routines, the costs of communication and coordination. On the other hand, all these elements are strategically manipulable by organizational actors, and in fact there is evidence that actors do exploit such strategic opportunities to maintain control of key resources and processes.

In a new born organization there are wide empty territories to occupy, and first movers have a good chance to win the gold race. The incentive system of a decentralized organization reinforces strategies of anticipation and sinking costs. Under such pressures, the system of local routines quickly crystallizes and prevents further change. 


\section{Fixing troubles}

\section{Fixing simple troubles}

Troubles range from very simple to very complex ones. Simple troubles fixing is usually an individual task, that reflects individual skills (although, as we shall see, these are embedded in the community links of the individual) and whose correct execution is mostly taken for granted. Thus, such simple troubles are a good starting point for describing capabilities that seem elementary both in terms of technical and social complexity.

One of the most common and simple troubles results in the inability of a single station to transmit a signal. The trouble is very easy to detect as it interrupts the whole station activity and it is also revealed by the automated control systems of the network. Thus, when a technician reaches the station whose transmission breakdown has been signaled, he is already told the type and possible causes of the trouble. In particular, if transmission breaks down totally, he must expect that something pertaining to the transmission system of the station (including a transmitter and a receiver) has been damaged.

In theory, since the transmission system is all integrated in a single board, the trouble could be easily fixed by changing the transmission board. This simplistic view is also the one encoded in the technical handbook provided by the technology supplier and adopted as the training platform for technicians. Thus, the formal standard procedure in case of such trouble is just "if a transmission error is signaled then change the board". However, even in this elementary case, the formal "official" knowledge is often misleading, and the experience of technicians has developed an alternative fixing routine. Failures of the standard procedure have created the ground for the emergence of the new routine. In fact, most technicians have soon learned that in many cases changing the transmission board would not eliminate the transmission breakdown. The reason is that there are interactions with other components of the technology that may result in a "transmission error" signal without directly concerning the transmission board itself. Instead of the official standard procedure, technicians have developed a more elaborate procedure which is grounded on a more complex diagnostic model of the trouble. The most surprising feature of the technicians' actual "expert" behavior in front of a transmission trouble is that their first intervention is rarely the transmission board substitution. Instead, they usually start resetting components of the station that affect indirectly the transmission process. The official standard procedure becomes only the last resort procedure when such preliminary tests have failed. This behavior is rooted in the model of trouble technicians have developed through collective experience. The model implies the belief that the transmission board technology is quite robust so it rarely fails, while other components are more fallible and have to be tested before. 
Thus, the implicit representation technicians use is not the simplistic one of the handbook but implies a more complex causal structure (see figure 2).

(a)

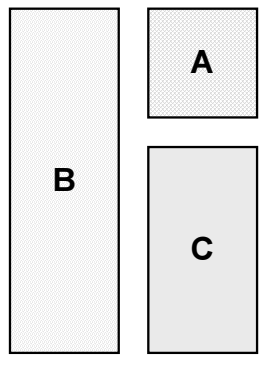

D

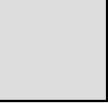

(b)
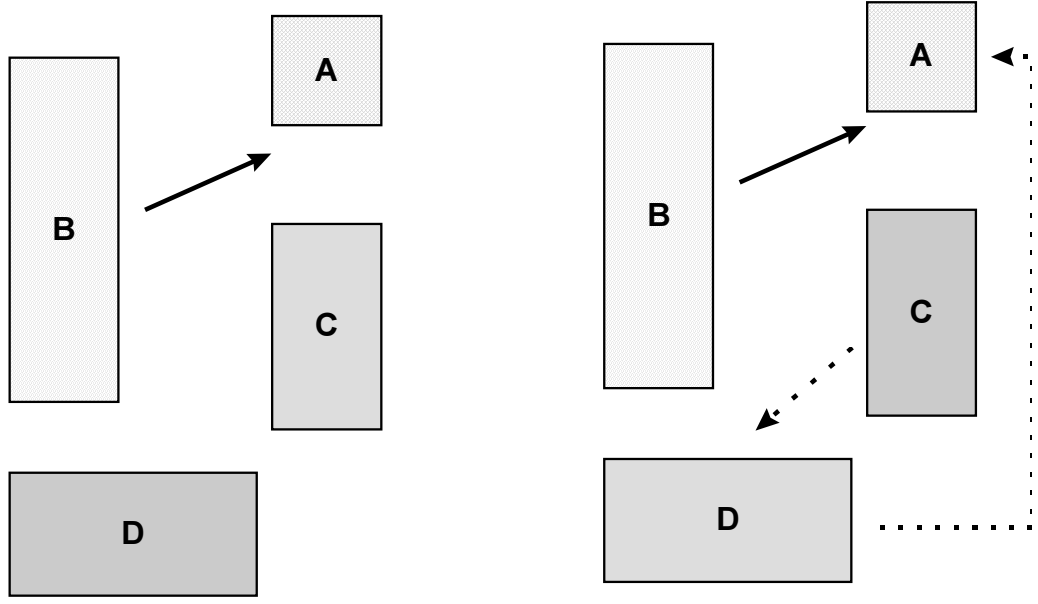

Figure 2. Fixing a trouble.

An alarm is signaled by the control system on board A. According to the control system (a) the trouble is localized in the board A. The BTS technicians' manuals sometimes suggest some alternative causes (b) for the problems, as for instance the board B. Nonetheless, on the bases of their field experience BTS technicians have learned that there are some more complex relationships among the boards and that the alarm on board A may be caused by some problems on board C.

A few remarks need to be made at this stage of our discussion.

- $\quad$ Such "enriched" representation is not derived from an explicit representation of the station inner working, but instead is induced from diagnostic experience: it is a diagnostic model of the test to be performed rather than a causal model of the technology. People learn this representation by calling more expert technicians, when a diagnostic impasse is experienced, and asking "what should I do then?" rather than asking which components are connected to the transmission board. We shall return to this remark more in depth later, but we can suggest that technicians tend to develop a model of their activity rather than a model of the technology they work on. 
- Individual technicians differ in terms of the diagnostic model they adopt. Differences are affected by the number of years of experience and membership to a particular subset of the whole technicians' "community of practice" (Lave and Wenger 1991; Seely Brown and Duguid 1991). For example, more experienced technicians differ from novice ones in terms of the number of possible causal links they test and degree of complexity they can handle with: the model of the trouble is usually richer in expert subjects. Also, expert technicians in different geographic areas may adopt different sets of causal links in their actual diagnostic activities. This seems to be closely related to the way expertise is accumulated and diffused. The enrichment of the diagnostic model is not only the outcome of individual experience with the technology, but to a large extent it is the result of communication among colleagues (Orr 1987). Communication sometimes arises as an ad hoc call in case of difficulties in trouble fixing, or as a "war story" told in the occasion of face to face encounters in the office, during lunch, or other meeting events (Orr 1990). Also, communication has tight boundaries defined by physical constraints (i.e. face to face meeting constrained by geographic distance) and social constraints (i.e. belonging to the same organizational unit). Thus, expertise is not only a matter of cumulated personal experience, but also a matter of membership to a community of practice within which experience is shared (a typically social dimension of expertise, thus, is the amount of people one personally knows and can call in case of need). For example, we have observed the case of an isolated technician, working in a physically remote area with low levels of integration within the community of colleagues, displaying more simplistic diagnostic activities despite having a long personal experience. This in turn has isolated him from colleagues, making him perceived as an "outgroup", further deepening his social distance from the work community and thus further impoverishing his personal skills.

\section{Testing alternative causes of a trouble}

Troubles can be more complex than that, however. Not all troubles have a repetitive and predictable nature. They often require a more subtle diagnostic task, involving the identification of the trouble nature and the construction of ad hoc sequences of tests identifying the appropriate repair operations. In those cases, the concept of routine as a mere behavioral pattern would be of little use to portray the technicians' activity. Nevertheless, the structure of diagnostic and repair activities shares important features with the one depicted in the example above. Another example will provide some illustration to this point.

One night, the automatized network control system was signaling that the fourth of the eight time slots managing multiple simultaneous calls in a station was affected by a $70 \%$ error (this means that communication in that time slot was totally flawed). One 
possible source of the error was of course a defect in the board containing the time slot itself. But, as in the former case, errors can result from troubles in coupled components. Two technicians had reached the site. No one directly thought to directly substitute the board containing the time slot (again, just like in the trouble fixing example described above), nor they started debating about the hardware structure. Rather, both technicians immediately contended about the appropriate representation of the diagnostic task. As we shall see, such representation carries only indirectly a representation of subsystems of the technology. The first object of debate was whether it was possible or not to perform a loop test, i.e. a simulation of a communication loop controlled by a portable computer. Once ascertained that the test could be performed, the first step was to replicate the error in the simulated context. The confirmation of the error was supporting the validity of the simulated call loop in reproducing the real trouble. Once this was established, the loop test structure was suggesting three possible sources of the error:

a) a default in the time slot

b) a default in the receiver sending inputs to the time slot

c) a default in both

This was because the loop test was passing messages through those two elements.

The implicit sequence of tests suggested by this representation was thus to eliminate possible alternative causes of error, selecting among the three possible sources (from a logical point of view, thus, the diagnostic test was similar to what psychologists of thinking call a selection task - Wason 1972).

The first test actually performed was to let the simulated call pass through another time slot connected to the same receiver. Since this alternative time slot was working well, it was logically clear that the receiver was not defective, and thus the only possible cause of error was a default in time slot four. However, the technicians didn't stop their test sequence, but performed all the sequence of test suggested by the loop test structure, repeating a loop test by coupling the fourth time slot to another receiver. Thus, technicians ignored the selective value of the first evidence and performed all the sequence despite the uselessness of the last test. This provides an interesting paradox. Technicians appear to follow a seemingly "automated" sequence of actions, but this happens in a context that they are experiencing for the first time and with a sequence that they are generating for the first time.

In order to interpret this paradox, it may be useful to look at the drawings one technician made to explain what he had just done to one of us (see figure 3a). The drawing was NOT representing the actual functional structure of the hardware (see figure $3 \mathrm{~b}$ ), with its connections among components, but instead was representing the logical structure of the loop test, connecting together components of the station 
hardware not through their actual physical links, but in their functional position within the test scheme (this brings to a physically false representation of the hardware, in facts). Thus, they built a model of their activity, not of the station structure. What seems to be routinized, thus, is not the sequence of actions itself (the series of tests) but how technicians derive a sequence of actions from the representation of the task they build. Within this representation, they ignore the evidence made available by experience until the sequence is entirely performed (like in the "batch" routine mode defined by Stinchcombe 1990). In other words, what seems to be routinized is the way they think through the representation and consequently act. The timing of their activities during this episode is revealing. It took them a while to identify the kind of test to perform but once this was made, the tests were performed in a very rapid sequence and in a seemingly "thoughtless" way.

\section{timeslots boards}

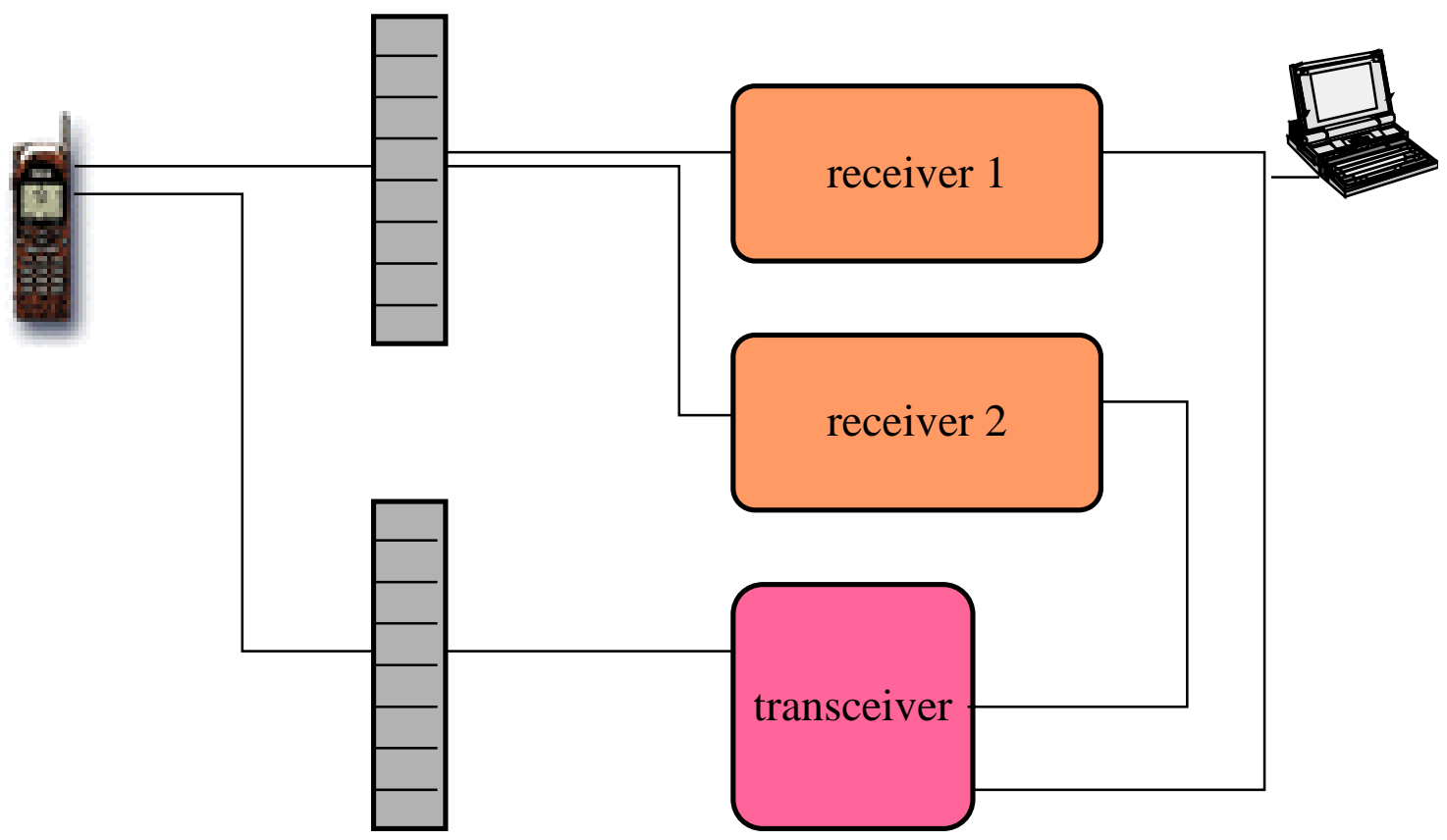

Figure 3a. Model of the trouble on the time-slots.

The representation of the hardware as drawn by a BTS technician is based on the structure of the test applied to reproduce the alarm. This model is different from a representation of the hardware shown in the next figure $(3 b)$. Here the technician draws twice the same 


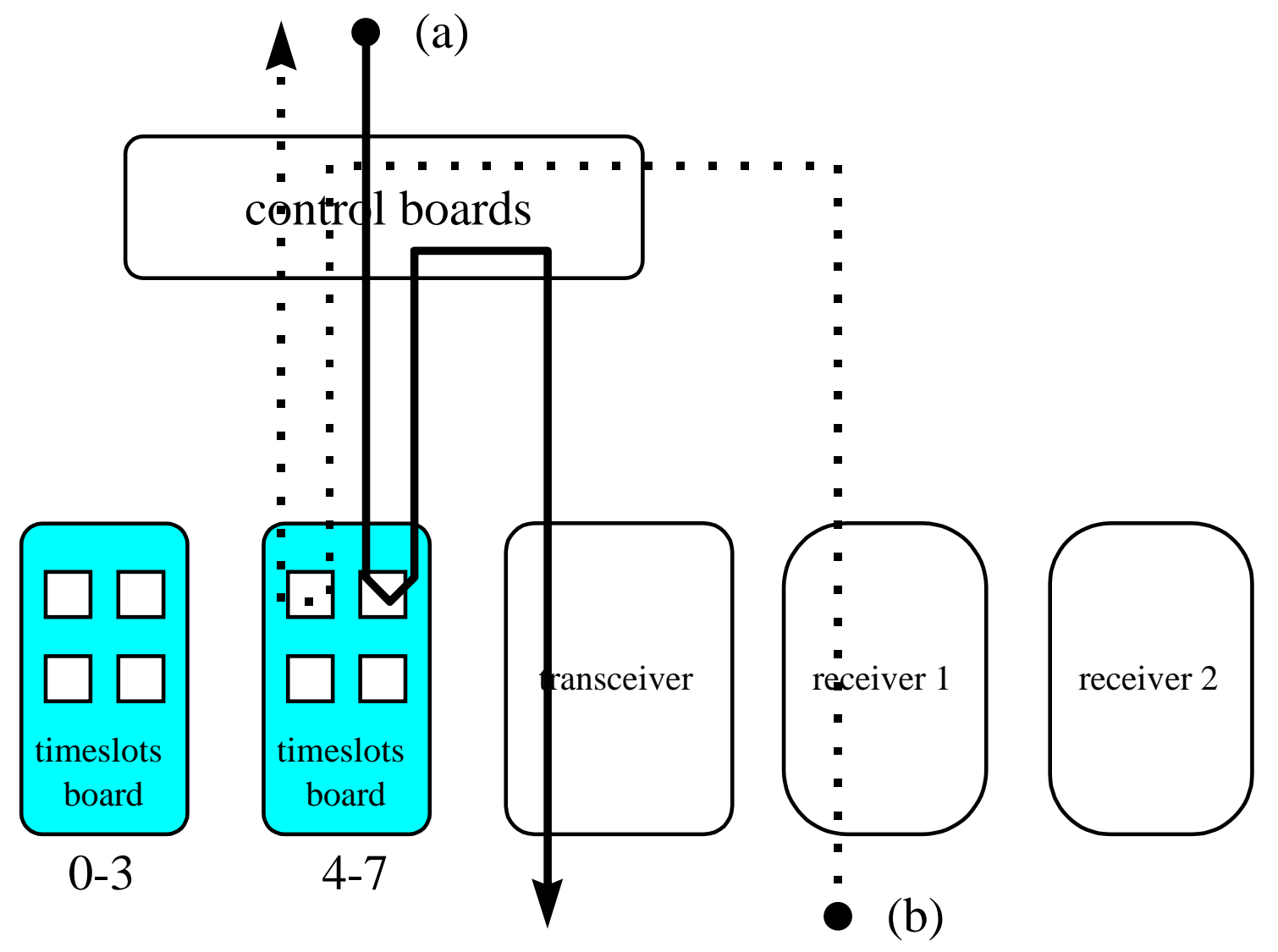

physically settled into two different boards.

Figure $3 \mathrm{~b}$. Scheme of the hardware structure involved the time-slot trouble.

Communications flows ( $a$ and $b$ ) are managed by a system of control boards that exchange signals with the transceiver and the receivers through the timeslots boards.

\section{Interpreting troubles}

Sometimes troubles are even harder to interpret. A station may stay out of operations for more than one day, without people being able to solve the trouble. In those cases interpreting the trouble becomes a highly social activity, since it usually involves the presence of many technicians and communications with an even larger 
number of them. In such cases, long discussions are held in the site, at lunch, or in phone conversations. We could observe many of those cases. When such "hard" diagnostic problems arise, the way people model and understand trouble fixing becomes even more relevant and an object of open discussion. Usually, after repeated testing activities fail, people start using "substitutes of direct experience" (March, Sproull and Tamuz 1991; Weick 1991) as sources of interpretation and new actions. In other words, people stop testing diagnostic hypotheses against the station and starts testing hypotheses against mental entities. Typically, this process takes two forms.

Testing hypotheses against models. The typical situation is one in which a test is proposed, and other technicians deny its validity on the ground of their model of the technology. This is the only case in which we have seen an explicit model of the technology emerging beyond the model of the test activity. Sometimes discussions can be so general that they concern not only the GSM technology, but broader notions of communication technology. This is not surprising given the higher level of abstraction and explicitness of such discussions: a sort of "academic" style emerges in these cases, leading to an explicit comparison of representations of how a station works. Since models of different scope and width are confronted, these are also opportunities in which individual representations are enriched and diffused in explicit form.

Testing hypotheses against case histories. In most cases, however, the discussion is less academic and resorts to analogous cases as sources of comparison and validation/discrediting for proposed interpretations. The usual format of those discussion is that a technician describes his difficulties in dealing with the trouble and his new hypotheses, and another technician starts a tale concerning situations he has faced that might provide useful analogies. Those tales are highly interactive, since they often imply questions and answers and the intervention of other persons. These tales closely resemble the "war stories" described by Orr (1987; 1990$)$. Interpretations are provided in the context of narratives of action, and they are tightly coupled to descriptions of the activity involved. They rarely present an abstract picture of the problem, but instead follow a plot dictated by the actual sequence of actions tried in those similar contexts. It is noteworthy that when such tale tellers are asked to join the station and try a sequence of tests, they reproduce the same sequence of actions they have been narrating. Sometimes this involves the repetition of tests that they know that had already been tried without success by former technicians. We find here again the emergence of "useless" tests that are nevertheless performed. The close tie to the narrative form of case telling suggests that useless tests are replied because they are part of the story - in other words, they act as "evoking steps" (March and Simon 1958) necessary to the technician to reconstruct in action the pattern experienced in past cases. 


\section{Wrapping up}

Trouble-fixing is not just an automatic execution of a behavioral repertoire, but implies reasoning through a model of the trouble - there is some flexibility in actions that is hardly amenable to a strict behavioral view of routines

The model of the trouble, however, is embedded in a model of the trouble- fixing task that ties together representations of the technology, diagnostic activities and repair actions.

Once such a model is active, technicians execute all the actions it suggests in a batch mode, substantially ignoring the feedback from experience until the sequence is completed.

Although trouble-fixing skills may look as individual ones, elements of the individual representation are acquired not only through direct experience, but also through social activities of communication and debate within the boundaries of a "community of practice". The content of such communication rarely takes the form of abstract, explicit representations of the technology, while most often such representations are embedded in tales of the activity.

Thus, there is a mixed evidence of routinization in a strict (behavioral) sense. In order to describe trouble-fixing activities, one needs to account for both automatic action and reasoning, and feedback from experience seems to be relevant at some levels (defining the trouble) and irrelevant at some other level (performing sequences of actions). We suggest that introducing an explicit distinction between representations and behavior may be helpful. People seem to reason through representations (although these are highly context-dependent, task-oriented ones) and act automatically on the basis of such representations. Furthermore, the diffusion of representations is not less relevant than the replication of behaviors - but representations are mostly diffused in ways that depend on the social and practical context.

\section{Building a network station}

As we have seen, trouble fixing routines are social in nature, but their execution is mostly an individual task ; however, technicians are also engaged in activities implying joint coordinated execution and a more complex division of labor. The network station installation and set up is the most prominent example of such more complex patterns of action.

Basically, the station installation and set up consists of three phases (see figure 4): 
- $\quad$ "Room-Ready" phase in which the physical location (walls, pylons, public utilities connections) is predisposed for hosting the electronic hardware of the station;

- $\quad$ "Commissioning phase" during which the hardware is installed, configured and tested;

- $\quad$ "Integration phase", during which the station is further configured and connected to the GSM network.

Each of these phases belongs formally to different units, both OPI internal units and/or providers ones. There are very detailed standard procedures, agreed between OPI and its main technology provider, prescribing accurately how phases should be sequenced in time and how they should be executed (a technician compared the almost pedantic nature of such instructions to the one of his first motorcycle manual, specifying even that "one should put the parking-bar down when he parks). In particular, such procedures prescribe a strictly sequential arrangement of the phases and of single activities, and a rigid division of labor between OPI units and the providers' ones..

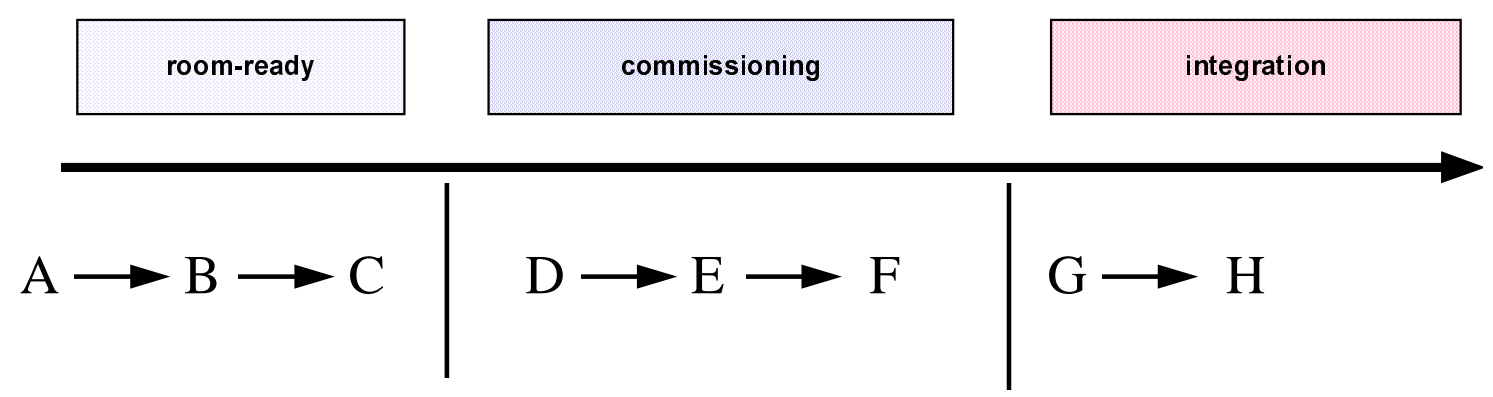

Figure 4. The standard operating procedure defined by OPI to build new BTS stations

Despite their very detailed structure, such standard procedures are in fact systematically violated in the field. Phases are overlapping, single activities are often executed in parallel, and the division of labor is re-arranged as a function of the timing of the activities. Moreover, OPI technicians participate to all phases (they shouldn't) and often substitute providers' technicians. Thus, the standard procedure is in fact "spontaneously re-engineered" by participants, generating an entirely different sequence of activities and division of labor (see figure 5). 


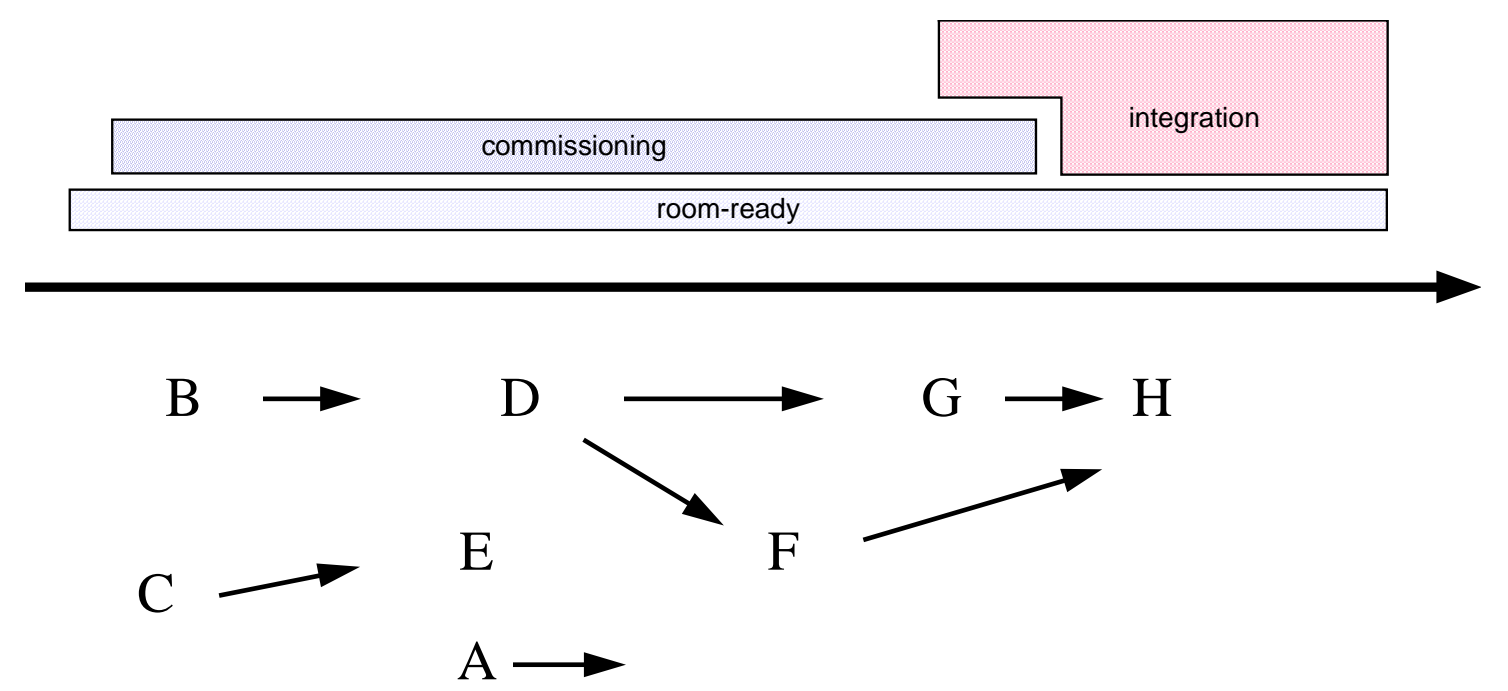

Figure 5. The emergent routine followed to build new BTS stations

The relevant point here is that such a re-organization violates the procedure in a peculiar way. In fact the official procedure is not "thrown away", but instead is used only for its descriptive value, while its prescriptive aspects are eluded. Paradoxically, it is just because the procedure is so detailed that activities can be restructured: participants tend to use the procedure as a check list of activities, whose official sequence can be ignored as far as their precise description allows to treat them as "modules" to re-assemble in practice (Pentland and Reuter 1994). In other words, the procedure allows a clear punctuation of the flow of activities, providing unambiguous labels for them, and thus provides a system of manipulable elements (Holyoak and Spellman 1993). Since the installation work has to be performed under a relevant time pressure (OPI had to quickly achieve a broad coverage of the national territory), activities that can be anticipated are consequently performed in parallel, reducing the cycle time. Some participants to the restructured routine that had previously worked on the same task with a different organization reported that in their former occupation, since activities were less clearly defined in highly detailed procedures, it was harder to subvert the customary sequence of actions - which implied a much longer cycle time. Thus, the formal procedure is not merely executed neither ignored, but instead it is used as a "structuring resource" (Suchman 1987) for manipulating the list of activities and restructuring their position in time.

Notice that the new arrangement of activities in time is not a rigid one: it is easily adapted to the specific contexts of each installation (e.g. to delays in some activity) and can be re-negotiated among the actors (for example, when a different providers' team is encountered, new arrangements on who should do what can be easily generated). We find here the same kind of flexibility that was found in the trouble fixing examples: once a representation of the problem is constructed, activities can be performed in 
routinary ways following the plan suggested by the representation. The usefulness of the standard procedure is in that it allows to easily build such representation in a shared and unambiguous way, so that a coherent division of labor can be established and single actions can be performed. In a way, what is strictly routinized is the execution of the single "activity modules," or chunks (Cohen and Bacdayan 1994). In turn, such a modularity is possible thanks to the descriptive power of the formal procedure.

The power of the modularity of routines is well shown by a subsequent example, which emphasizes the recombination possibilities (Nelson and Winter 1982; Zander and Kogut 1995) modularization provides.

After their first installation, some stations had to be empowered by the add-on of further boards for the reception and transmission of phone calls ("oversizing"). This was a somehow new activity, since it implied the restructuring of pre-existing installations. The main novelty was constituted by something like an "open heart" surgery over a working station. Furthermore, this activity had to be performed under a severe time constraint.

The structuring of the new activity was not procedurally specified by Head Quarters, but instead was delegated to team leaders. The new routine took shape through initial pilot oversizing experiments in a few stations where a group of technicians participated - after those early experiences, each technician could perform it individually. The main problem was that oversizing had to be performed by technicians without any direct feedback on the success of the operation. They were executing a kind of package of tasks whose effect could only be verified by the subsequent activation by a different organizational unit (the radio frequency technicians), often with some days of delay. This mix of novelty, time constraints and lack of feedback was creating a strong sense of anxiety in technicians. Facing this uncertainty about the outcomes and in the absence of an official procedure, technicians heavily resorted to past experience as a source of structuring for the new activity. The activity was not redesigned from scratch, but instead was organized though a new recombination of single "building blocks" of the installation routine. This way, technicians attention was focused on the few entirely new operations and on the connection among different building blocks, leaving unchanged the execution of the building blocks themselves, that were performed in an automatic way and were somehow taken for granted. The sequence of building blocks was heavily driven by the characteristics of the hardware. Thus, the correctness of operations was only assured to network technicians by the correct execution of a sequence of familiar tasks. Being unable to evaluate the outcomes, they had to rely on the process (March 1994). 


\section{Wrapping-up}

The emergence of the new installation routine shows how establishing a new routine may imply redefining the division of labor among actors and the distribution of knowledge among them.

The story shows the paradoxical power of formal rules and procedures in facilitating the coordination of such process. By supplying labels that punctuate the flow of activities and provide a shared representation of the task, the standard procedures act as descriptive resources that allow to violate their own prescriptive implications. BTS technicians are able to manipulate the formal representation to achieve a different sequence of actions, stressing once more the need to distinguish representations from expressions.

The punctuation of activities enhances the modularity of the routine, facilitating the architectural restructuring of the same set of building blocks or their selective recombination in a new routine (oversizing). Within single modules, actors are able to act in a semi-automatic way even in front of novel situations.

Furthermore, the familiarity of the single building blocks recombined in an unfamiliar task allows to use process feedback (the correct execution of each module) as a substitute for outcome feedback, guiding behavior in front of substantive uncertainty about the success of a sequence of actions.

\section{Coordination and hierarchy: The emergence of team leadership}

Coordination processes are at the core of organizational routines. The multi-actor nature of routines implies that individual behaviors have to be matched in order to ensure a smooth flow of actions. In complex action settings, coordination not only is part of virtually any routine, but becomes a specific process, connecting and interfacing single routines through communication, scheduling, task assignment and other related activities. In other words, coordination processes become crucial elements of the architecture of systems of interdependent routines.

A remarkable feature of the evolution of coordination practices in the technicians' organization is the emergence of an unplanned coordination structure ending up in the institutionalization of a formal hierarchy. At the beginning of our field observations, the technicians structure was a quite flat one: there were three to four teams per geographic area (the "zones"), within which roles were homogeneous, under the global responsibility of a zone manager. The team was explicitly designed as a group of peers, where any member of the team had to be on a same footing and cover equivalent functional roles., developing equal skills. At the end of our observations, after about 
one year, teams had developed a hierarchical structure, with the emergence (and subsequent formalization) of team leaders that had assumed coordination tasks as their almost exclusive activity. The process through which this hierarchical structure emerged is worth telling.

In the early months of activity, technicians field activity was still performed by small groups (usually pairs), and a significant amount of technicians' time (almost $50 \%$ ) was jointly spent in the office, where a rich amount of many-to-many communication flows was taking place through conversations, meetings and all sort of informal communication practices within each team. Scheduling and task assignment were performed through collective decision making in meetings at the beginning of the week or in ad hoc occasions. In fact, most coordination was not perceived as a per se process, but was instead immersed in the flow of ongoing activity. This practice was coherent with the original organizational design, that was explicitly emphasizing the homogeneity of individual competencies and roles within each team.

However, since the beginning it was clear that there were emerging pressures that might disrupt the planned homogeneity of team roles. Those pressures were originated by four main processes:

1. The number of technicians per zone was rapidly increasing.

2. The number of stations was increasing more rapidly than the number of technicians (from 8 to 20 per technicians in a few months), causing a shift from joint activity to individual activity on single stations.

3. Stations were installed increasingly far from local offices, increasing the time spent out of the office itself.

4. The amount of documentation to be produced by technicians was rapidly growing.

As a result, there was an increasing coordination need for scheduling activities during the week, assigning tasks, signaling unexpected troubles, filling reports on executed tasks. The dramatic decrease of time spent together in the office (from about half the working time to a few hours a week) was forcing the creation of communication nodes that might efficiently substitute for direct, many-to-many communication (Simon 1981)). Moreover, it was increasingly difficult for zone managers to communicate with all team members. The shift from a full networked to a star topology of communication was thus almost unavoidable. However, there are some less obvious features of this process.

In contrast with the official "team of peers" philosophy, those central communication nodes were quickly steadily occupied by a single person. Such a person was emerging from the team on the basis of quite occasional differentiation elements. In 
some teams, it was the one with more seniority. In other teams, it was the one which was controlling critical resources for bureaucratic work, like a computer. In other cases, it was the one having acquired more status symbols, like the use of the company car. The key point is that no one of those distinctive elements was officially recognized as relevant - each technician had to be like the others - nor was it based on major competencies related to the technicians core task. But as such distinctive elements were making a team member easier to recognize from others, it was creating a spontaneous "reference" for hierarchy that was quickly acquiring centrality in the communication flow. In other words, although zone managers were denying any difference among technicians, they were quickly recognizing elements of differentiation and using them for creating gate-keeping positions. Initially, labels of "team leader" were utilized, but under the premise that team leadership would not imply any differentiation in task or resource allocation - team leaders had to be "primi inter pares"). However, soon first among peers became first among unequals.

Once this initial (and, as we have seen, based on quite accidental criteria) differentiation was established, it was creating a sort of monopoly over communication resources. To provide just an anecdote, at the beginning the team leader in one zone was just the most able in using the laptop where some critical software was residing. Subsequently, being the best one in using it he was keeping it with himself most of the time. Finally, a password was creating a de facto monopoly in its use and was inhibiting others from filling the competence gap.

As coordination tasks were increasingly loading team leaders activity, team leaders were starting to spend more time in the office than others, thus reducing their direct involvement in field operations. Once more, this was contradicting in facts the official policy on teams. When, talking to managers, we were remarking that there was an increasing pressure towards the specialization of team leaders, they were strongly denying the desirability of such specialization. Nevertheless, they were reinforcing it in practice, for example increasing the reporting tasks of team leaders over team activities. In fact, the responsibility for communication was becoming implicitly a responsibility for activity, and the team leader was increasingly asked to be responsible for the outcomes of the team activity. This implicit shift from communication gate-keeping to accountability for the team performance was the key passage in establishing hierarchical roles.

A related process is that as specialized coordination activities are created, they tend to bootstrap on themselves: for example, a team leader has less time to visit the field because he needs to spend time in creating software that will help him in dealing with the existing software, or in creating procedures that he will have to manage. Coordination recursively generates coordination needs. The process is very similar to the one observed in studies of managerial work (Mintzberg 1973, Hannaway 1989). 
The process through which the gap between practice and formal prescriptions has been filled is an example of "crawling institutionalization" of practice. The emerging hierarchical structure of team coordination has not been recognized through a single, deliberate decision, but instead has been frozen into a series of formal devices and acts.

For example, the emerging hierarchy has been progressively embedded in administrative software. There has been an increasing number of applications in which team leaders have writing access, while technicians only have reading access.

More generally, procedures started making explicit differences between team leaders and the others. For example, procedures regulating night availability of technicians had been revised to explicitly exclude team leaders from night shifts.

Finally, the process of institutionalization of team leadership has reached its achievement when team leaders have been formally relieved of field operations (e.g.: maintenance or trouble fixing). This exemption makes the final symbolic detachment of coordination activities from the field, separating the physical and social territory of the team from the one of its leader.

\section{Wrapping-up}

Thus, this picture of the evolution of hierarchy in coordination processes keeps some classical flavor, but adds some new ingredients. On the one hand, it clearly reflects pressure of the growing task complexity on communication structures, that creates opportunities for their increasing centralization (Simon 1981). On the other one hand, the way such opportunities are seized and the dynamics of the process of centralization suggest a few additional features.

- Hierarchical roles seem to evolve out of quite occasional initial differentiation elements. In particular, those elements of differentiation provide initial cues (tags: see Holland 1995) helping the management to select gate-keeping persons in the communication process.

- The amplification of such initial differences into stable hierarchical roles is not only favored by the pressure of task complexity, but is also sustained by selfreinforcing processes, driven by the acquisition of critical resources (Coriat and Dosi 1994) by team leaders and by some recursive, bootstrapping features of the coordination work.

- The most relevant outcome of such process is the progressive enucleation of coordination activities from ongoing activities. Once these coordination activities are singled out, they cease to be coupled to single persons and become stable elements of the architecture of the system. Thus the emergence of hierarchy 
restructures the system of routines, changing the way they are interfaced and aggregated in higher level patterns of activity.

- Finally, "organizational artifacts" such as software, procedures and job descriptions are the vehicles through which emerging hierarchical roles are formalized and some irreversibility is introduced in the process.

\section{Concluding remarks}

Inferences one can draw from a case study are highly local and, in many senses, subjective. What we have learned is most of the time how the concept of routine should be articulated in order to allow us to tell our story. A different story might require a different "unbundling" of routines, and different observers might tell differently the same story. The main implications of our case study can be usefully summarized by starting from the definition of routines in their narrow sense, and suggesting in which directions we had to enrich it to describe what we have observed

Sid Winter has recently defined routines in the narrow sense as:

complex, highly automatic (and at least in that sense "unconscious") behaviors that "function as a unit" and typically involve high levels of information processing that is largely repetitive over separate invocation of the routine. (Cohen et al. 1996, p.663)

If one looks at the way key capabilities (such as installation or trouble fixing) are learned and stored by network technicians, routines in their narrow sense are only a part of the picture, and probably not the most relevant. To be sure, there are many examples of automatized, tacit behaviors in our story. But all of them are embedded in more complex patterns of action in which interpretation, reasoning, more or less explicit manipulation of mental representations, deliberation and design take a relevant part. Routines in the narrow sense are important, but they don't suffice in any case to sustain a reasonable performance in activities of strategic relevance to the firm.

More broadly, a "behavioral" view of routines seems of little use to tell our story. We rarely found highly repetitive behavior. In general, a certain degree of flexibility seems to be a relevant component of skilled action, both at the individual and the collective level. The single technician dealing with a trouble fixing task or a team dealing with a new station installation rarely repeat the same behaviors, and are usually able to adapt to different situations with different behaviors. In turn, those different behaviors are not apparently drawn from a given behavioral repertoire, but come out of an interpretive activity that implies some manipulation of a representation of the task at hand. 
The most economic way to account for the interplay between such interpretive activity and actual behavior is to make a distinction between representations and expressions (Cohen et al. 1996). We suggest that our technicians store representations of the task under the form of mental models, i.e. a reduced scale model of the external reality and of available actions (Craik 1943; Johnson-Laird 1983; Holland et al. 1986), and construct a model of the problem at hand by retrieving such representations and combining them into new ones. Once this representation is achieved, it suggests appropriate behaviors (expressions) that usually exhibit properties of modularity, tacitness and high automaticity. Thus, "routines in the narrow sense" are often found at the lower level of action patterns, as "behavioral modules" that are activated and assembled following the definition of the problem that has been reached. Automated sequences of tests in trouble fixing or elementary action modules in installation are examples of routines in the narrow sense that are recurrent in our observations. Our point is that they cannot be understood without resorting to a broader architecture of skills within which mental models play a key role.

The term "mental model" may be misleading if it suggests that representations are only stored in people's minds (Norman 1991). In fact, we have shown that many representations are stored in people's mind and in artifacts such as procedures, software, or physical features of the environment that are resources structuring mental models of individuals and teams (Hutchins 1991): see the example of how detailed procedures help to spontaneously re-engineer the installation process. It is a relevant matter of fact that design can shape the emergence of routines through such artifacts.

Looking at representations not only helps to account for behavioral flexibility, but makes it easier to understand how capabilities are stored and diffused within the organization. Although there is evidence of processes of direct imitation of behavior, most diffusion processes are vehiculated by the explicitation of models of the task, as we have seen for trouble fixing activities. Needless to say, representations are diffused in context-dependent forms (Holyoak and Spellman 1993), rather than in abstract ways. Technicians suggest to each other new causal connections among components or interpretations of the technology in the context of sequences of tests or in narrative forms, and the availability of evoking inputs in task-related descriptions of the trouble are critical in eliciting colleagues' representations. Nevertheless, there is little doubt about the fact that "replicators" in the process of diffusion are often (incomplete) representations rather than expressions (behaviors). External representations embedded in artifacts play a similar role in storing and replicating productive knowledge, as we have seen above.

The nature of diffusion/replication processes is especially relevant in defining how individual and collective competencies are interrelated, and hence in bridging the gap between individuals and collective entities such as groups, organizational units or firms at large. Nelson and Winter (1983) heavily draw on the analogy between individual 
skills and routines. Our observations support such analogy, but suggest that more attention should be devoted to the complicated interrelationships between the individual and the collective level. The case study highlights three elements of special relevance.

1. On the one side, processes of diffusion help to frame individual skills in the context of collective activities. Although many of the competencies we have observed are ultimately individual (they are not "distributed" among actors), like in trouble fixing activities, they are developed and maintained within a community of actors whose boundaries, structure and communication patterns deeply affect what individuals know. In this peculiar sense, individual skills are organizational phenomena.

2. Moreover, the diffusion of competencies within groups of actors rely on a shared language that enables to make representations communicable (Hutchins and Hazelhurst 1990). Such shared language is an organizational construct, and it interfaces individual skills and genuinely collective capabilities, which are distributed among participants.

3. Artifacts provide structuring resources (Suchman 1987) that are shared by actors. We have seen how the hardware and software used for diagnostic activity provide both a common representation of the task and suggest sequences of actions (Hutchins 1995).

Finally, organizational capabilities are not mere collections of routines (however defined, narrowly or broadly). As far as there are complementarities among routines, some form of architectural coherence among them is needed to achieve efficiency and coordination. For example, the initial patchwork of competencies generates locally coherent "work styles" at the zone level. We have also seen how the coordination architecture of a system of routines evolves into a hierarchical structure, extracting coordination processes out of single routines and transforming coordination into a per se activity attributed to a specialistic, hierarchical role. We have also seen how in both examples the architecture of systems of routines is shaped by the pressure of "functional" needs (exploiting complementarities among them) but also by strategic moves of actors motivated by local interest (Coriat and Dosi 1994). We suggest that further exploring issues of complementarities among routines and architectural coherence would considerably enlarge our understanding of organizational capabilities. 


\section{References}

Amit, R., Schoemaker, P.J.H., 1993, Strategic Assets and Organizational Rent, Strategic Management Journal, 14, 33-46.

Barney, J., 1991, Firm Resources and Sustained Competitive Advantage, Journal of Management, 1, 99-120.

Baum, J.A.C., Singh, J.V., 1994, (eds.), Evolutionary Dynamics of Organization,

Cohen, M.D., 1991, Individual learning and organizational routines: emerging connections, Organization Science, 1, 135-139.

Cohen, M.D., Bacdayan, P., 1994, Organizational routines are stored as procedural memory: Evidence from a laboratory study, Organization Science, 5, 545-568.

Cohen, M.D., Burkhart, R., Dosi, G., Egidi, M., Marengo, L., Warglien, M., Winter, S., 1995, Routines and Other Recurring Action Patterns of Organizations: Contemporary Research Issues, Industrial and Corporate Change, 5 (3), pp: 653698.

Coriat B., Dosi G., 1994, Learning How to Govern and Learning How to Solve Problems. On the Co-evolution of Competences, Conflicts and Organizational Routines, IIASA Working Paper, Laxenburg, Austria.

Craik, K., 1943, The nature of explanation, Cambridge University Press.

Egidi, M., Narduzzo A., 1997, The emergence of path-dependent routines in cooperative contexts, International Journal of Industrial Organization.

Gersick, C., Hackman, J.R., 1990, Habitual Routines and task performing groups, Organizational Behavior and Human Decision Processes, 47, 65-97.

Hannaway, J., 1989, Managers managing: the working of an administrative system, Oxford University Press.

Holland, J.H, 1995, Hidden Order: How Adaptation Builds Complexity, Addison Wesley.

Holland, J.H, Holyoak, K.J., Nisbett, R.E., Thagard, P.R., 1986, Induction, The MIT Press. 
Holyoak, K.J., Spellman, B.A., 1993, “Thinking” in Annual Review of Psychology, 44, 265-315.

Hutchins, E., 1991 'The social organization of distributed cognition', in Resnick, L.B., Levine, J.M., Teasley, S.D., (eds.) Perspectives on Socially Shared Cognition, 283-307, American Psychological Association.

Hutchins, E., 1995, Cognition in the Wild, The MIT Press.

Hutchins, E., Hazelhurst, B., 1990, Learning in the Cultural Process, in Langton et al. (eds.) Artificial Life II: Studies in the Sciences do Complexity.

Johnson-Laird, P.N., 1983, Mental Models, Harvard University Press.

Kogut, B., Zander, U., 1992, Knowledge of the Firm, Combinative Capabilities, and the Replication of Technology, Organization Science, 3, 383397.

Lave, J., Wenger, E., 1991, Situated Learning, Cambridge University Press.

Levinthal, D., Warglien, M., 1997, Landscape design: designing for local actions in complex worlds, CEEL WP.

March, J.G., 1994, A primer on decision making: how decisions happen, The Free Press.

March, J.G., Simon, H.A., 1993, Organizations, 2nd ed. (1st ed. 1958), Blackwell.

March, J.G., Sproull, L.S., Tamuz, M., 1991, Learning from Samples of One or Fewer, Organization Science, 2, 1, 14-39.

Mintzberg, H., 1973, The Nature of Managerial Work, Harper \&Row.

Nelson, R.R., Winter S.G., 1982, An Evolutionary Theory of Economic Change, Belknap Harvard Press.

Norman, D.A., 1991, Cognitive artifacts, in J.M., Carroll (ed.) Designing interaction: Psychology at the human-computer interface, 17-38, Cambridge University Press.

Orr, J., 1987, Narratives at Work: Story Telling as Cooperative Diagnostics Activity, Field Service Culture, June, 47-60.

Orr, J., 1990, Sharing Knowledge, Celebrating Identity: War stories and Community Memory in a Service Culture, in Middleton, D.S., Edwards, D., (eds.) Collective Remembering: Memory in Society, Sage Publication. 
Pentland, B.T., Rueter, H.H., 1994, Organizational Routines as Grammars of Action, Administrative Science Quarterly, 39, 484-510.

Seely Brown, J., Duguid, P., 1991, Organizational Learning and Communities-of-Practice: Toward a Unified View of Working, Learning, and Innovation, Organization Science, 1, 40-57.

Simon, H.A., 1981, The Sciences of Artificial, The MIT Press.

Staw, B.M., Ross, J., 1987, When to pull the play, Harvard Business Review, feb.

Stinchcombe, A.C, 1965, Social structure and organization, in March, J.G., (ed.), Handbook of organizations, Rand McNally.

Stinchcombe, A.L., 1990, Information and Organizations, University of California Press.

Suchman, L., 1987, Plans and situated action, Cambridge University Press.

Teece, D., 1984, Economic Analysis and Strategic Management, California Management Review,26, 3, 87-110.

Teece, D., (ed.), 1987, The Competitive Challenge, Strategies for Industrial Innovation and Renewal, Ballinger.

Weick, K.E., 1979, The social psychology of organizing, 2nd ed., AddisonWesley.

Weick, K.E., 1991, The nontraditional quality of organizational learning, Organization Science, 2, 1, 41-73.

Wernerfelt, B., 1984, A Resource-based Theory of the Firm, Strategic Management Journal, 5, 171-180.

Winter, S.G., 1986, The research program of the behavioral theory of the firm, in Gilad, B., Kaish, S., (eds.), Handbook of Behavioral Economics, 151-188.

Winter, S.G., 1987, Knowledge and competence a strategic assets, in Teece (ed.), The Competitive Challenge, Strategies for Industrial Innovation and Renewal, Ballinger. 